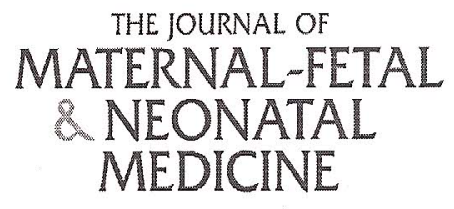

hrtp://informahealthcare.com/jmf ISSN: $1476-7058$ (print), 1476-4954 (electronic)

J Matern Fetal Neonatal Med, Early Online: 1-5 (c) 2013 Informa UK Ltd. DOl: 10.3109/14767058.2013.845158 informa

healthcare

ORIGINAL ARTICLE

\title{
Acupuncture in post-date pregnancy: a pilot study
}

\author{
Isabella Neri, Francesca Monari, Chiara Salvioli Midwife, and Fabio Facchinetti
}

Mother-Infant Department, University of Modena and Reggio Emilia, Modena, Italy

\begin{abstract}
Aim: Pharmacological labor induction is obtained through intracervical/vaginal prostaglandins and/or oxytocin infusion; however, the use of these agents produces fetal and maternal side effects. Traditional Chinese medicine advocates the use of acupuncture to soften the cervix and induce uterine contractions. The aim of the present study is to investigate the effect of acupuncture to induce labor. Acupuncture was applied in post-date pregnancies one week before a planned induction; the primary outcome was the rate of women submitted to labor induction for prolonged pregnancy at week $41-5$.

Methods: After informed consent, 221 undelivered women ranging between $40 \div 2 / 40+6$ gestational age were considered eligible for the study and 202 were randomized to receive acupuncture or observation. Sessions of acupuncture were planned every odd day from the randomization till 41 a week plus 4 days. At $41+5$ week a pharmacological induction of labor was planned.

Results: The total rate of labor induction did not significantly differ between observation and acupuncture group (20\% versus 17\%). Moreover no differences were found as far as the indications to induce labor, in particular "prolonged pregnancy" was similar between groups (8/96 versus 5/99). To investigate between-group differences in time elapsed between inclusion and delivery, survival analysis was performed excluding women requiring labor induction: women receiving acupuncture showed a trend to deliver earlier than women in the observation group $(p<0.09)$.

Conclusion: The present study demonstrated that acupuncture applied every odd day for one week seems ineffective in reducing the rate of labor induction performed for prolonged pregnancy at $41+5$ weeks. Previous reports reached similar conclusions, independently of the different timing, duration and mode of stimuli application.
\end{abstract}

\section{Keywords}

Acupuncture, labor induction, post-date pregnancy

\section{History}

Received 9 July 2013

Accepted 12 September 2013

Published online 28 October 2013

\section{Introduction}

The management of prolonged pregnancy and expectant versus elective induction of labor remains a controversial issue among obstetricians. A US trial involving induction after 41 weeks gestation compared with awaiting spontaneous labor was associated with a lower rate of perinatal mortality and morbidity [1].

Pharmacological labor induction is obtained through intracervical/vaginal prostaglandins and/or oxytocin infusion; however, the use of these agents produce fetal and maternal side effects [2]. Namely, oxytocin increases the rate of intervention in labor by caesarean section (c.s.) and operative delivery [3]. On the other hand, vaginal prostaglandin administration increases the risk of an excessive uterine activity and consequent fetal distress without affecting the rate of c.s. [4].

Address for correspondence: $\mathrm{Dr}$ Isabella Neri, Mother-Infant Department, University of Modena and Reggio Emilia, Via del Pozzo 71, 41100 Modena, Italy. Tel: 059-4222659. Fax: 059-4224394. E-mail: isabella.neri@unimore.it
To avoid such side effects, non-pharmacological methods of labor induction, including acupuncture, have received attention. Traditional Chinese medicine advocates the use of acupuncture to soften the cervix and induce uterine contractions [5]. Although several Chinese studies have been published on the topic, they unfortunately do not fulfill the scientific criteria of a randomized controlled trial.

The more recently updated Cochrane Review, which includes western studies, concluded that the effectiveness of acupuncture applied during the last month of pregnancy for spontaneous labor onset remains to be demonstrated due to insufficient data. Indeed the final analysis was made on a single report that included 54 women, and the results were not incorporated in the review due to methodological bias [6]. Two subsequent studies carried out in prolonged pregnancy did not clarify the issue, with results showing disparate conclusions $[7,8]$.

Previous studies do not allow a working hypothesis on the rate of effectiveness of acupuncture to favorite labor onset. The present randomized, controlled study represents a further extent to investigate such issue. Acupuncture was applied in post-date pregnancies one week before a planned induction. The primary outcome was the rate of women submitted 


\section{I. Neri et al.}

to labor induction for prolonged pregnancy at week $41+5$. Secondary outcomes were the rate of induction for other indications and the length of first and second stage of labor.

The study was recorded at the web site ClinicalTrial.gov with the number NCT01052857, "Efficacy of acupuncture in induction of labor".

\section{Materials and methods}

Pregnant women at term referred to the Midwife-Led Delivery Unit at the Mother-Infant Department of the University of Modena and Reggio Emilia were eligible for the study. The trial was recorded using the Protocol Registration System of the FDA at the website ClinicalTrials.gov and approved by the Institutional Review Board.

Inclusion criteria were: low-risk singleton pregnancy, intact membranes, planned delivery at the Unit and gestational age ranging between $40+2$ and $40+5$ weeks, as estimated by ultrasound before the 12 th week. Exclusion criteria were: maternal disorders, previous uterine surgery, contraindications to vaginal delivery, and Bishop score $\geq 6$.

After informed consent, undelivered women were randomized to acupuncture or observation. A computer-generated randomization list, in which odd and even numbers allocated patients to observation or active treatment, was employed. A senior Midwife disclosed the list for the next treatment allocation.

Fetal surveillance was planned (Figure 1) including serial non-stress-test and amniotic fluid index (AFI) evaluations. According to local guidelines, pharmacological labor induction was indicated for prolonged pregnancy reaching $41+5$ weeks, pre-labor rupture of membranes lasting $>18$ hours in absence of labor onset, or Amniotic Fluid Index $\leq 4$.

The acupuncture session (performed by a licensed acupuncturist, I.N.) consisted of the insertion of sterile, disposable $0.30 \times 4 \mathrm{~mm}$ acupuncture needles (Huanqiu, Qiu Tian, S.Marino); after reaching the $D e Q i$ sensation, needles were left in situ for 30-40 minutes. During the session women were positioned in a right lateral position in a comfortable, quiet room.

Figure 1. Fetal surveillance protocol in post-date pregnancy which includes serially non-stress test (NST) and amniotic fluid index (AFI) evaluation.
I Matern Fetal Neonatal Med, Early Online: $1-5$

Following Traditional Chinese Medicine (TCM) indications it has been stimulated acupoints able either to enhance uterine activity and ripe the cervix or to relax the muscles and reduces anxiety [3]. Hegu Large Intestine 4 is located in the middle of the first interosseous of hand and the needle is inserted of a depth of 1-2 cun It is claimed to improve uterine contractions.

Sanyinjiao Spleen 6 is located 3 cun above the medial malleoli and the needle is inserted to a depth of 1.5-2.5 cun. It is claimed to promote the ripening of the cervix and to promote uterine contractions.

Zhusanli Stomach 36 is located 3 cun above the tip of the medial malleolus on the medial border of the tibia and the needle is inserted to a depth of 1-2 cun. Such point is able to improve the circulation in the pelvic organs and employed in several acupuncture formula.

Yanglingquan Gold Bladder 34 is located in the depression anterior and inferior to the head of the fibula and the needle is inserted to a depth of $1-2 \mathrm{cun}$. Such point is indicated for the relaxation of muscles and tendons and employed in several acupuncture formula. It has been also described an effect on the cervix ripening when associated to the Thaichong Liver 3.

Thaichong Liver 3 is located in the foot, in the interosseus between the first and second toe and the needle is inserted to a depth of $0.2-0.5 \mathrm{cun}$. It is employed in particular to resolve the liver $Q i$ stagnation.

Neiguan Pericardium 6, located on the palmar side of the forearm 2 cun above the transverse crease, and Shenmen Heart 7 , located on the outer side of the wrist, between the ulna and the pisiform bones, were also added to the protocol and applied in women manifesting feelings of fear or anxiety.

Sessions of acupuncture were planned every odd day from the randomization till 4 la week plus 4 days. At $41+5$ week a pharmacological induction of labor was planned.

The $t$ test was applied to compare continuous variables and $x^{2}$ was applied to compare relative frequencies. Data are reported as Mean \pm Standard deviation $(\mathrm{M} \pm \mathrm{SD}$ ). A $p$ value lower than 0.08 was considered as significant.

Kaplan-Meier survival analysis to investigate betweengroup differences in time elapsed between inclusion and delivery was also performed.

\section{OBSERVATION}

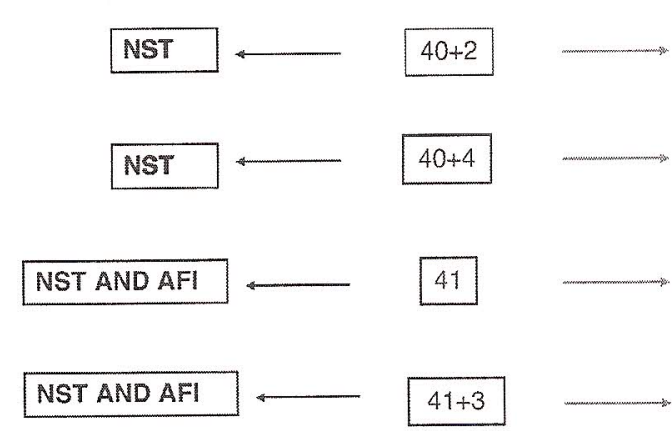

ACUPUNCTURE

NST ACUPUNCTURE SESSION

NST

ACUPUNCTURE SESSION

NST AND AFI

ACUPUNCTUME SESSION

NST AND AFI

ACUPUNCTUPE SESSION 


\section{Results}

The flow chart of patients inclusion is reported in Figure 2. Two women dropped out in the acupuncture group and five in observation group due to low compliance; thus, the final analysis was based on 195 cases. No differences were observed between the observation and acupuncture group in terms of maternal age $(32.4 \pm 4.1$ versus $33.1 \pm 4$ years $)$, rate of nulliparous women ( $77 \%$ versus $74 \%$ ), pre-pregnancy body mass index (BMI) $(23.5 \pm 3.5$ versus $24.4 \pm 3.5)$ and gestational age at study entry $(282.3 \pm 0.7$ versus $282.7 \pm 0.9$

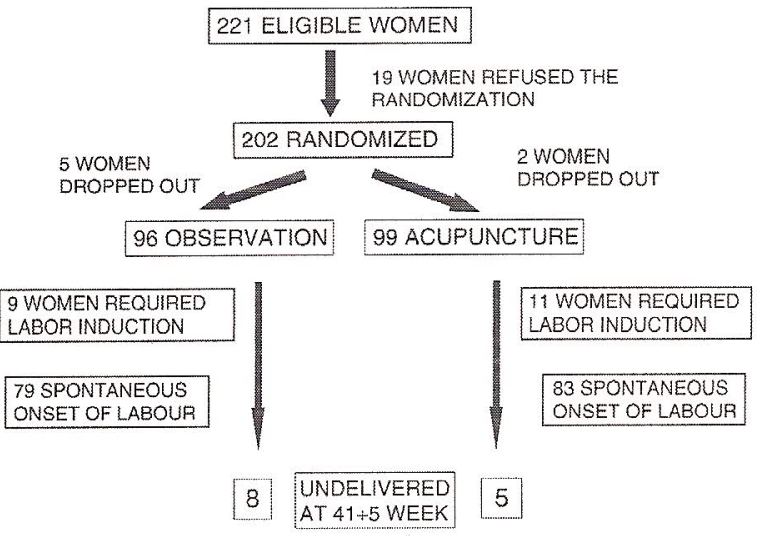

Figure 2. Flow chart of the trial. days). The rate of graduate ( $41 \%$ versus $46 \%$ ) and employed women $(92.6 \%$ versus $92.8 \%)$ also did not differ between the acupuncture and observation group.

In the observation group labor induction was required for pre-labor rupture of membranes lasting $\geq 18$ hours in absence of labor onset (two cases) and for AFI $\leq 4$ (seven cases) Among the remaining 87 women, 79 experienced a spontaneous onset of labor and eight remained undelivered at $41+5$ weeks, requiring labor induction for prolonged pregnancy.

In the acupuncture group labor induction was required for pre-labor rupture of membranes (six cases) and for $\mathrm{AFI} \leq 4$ (five cases). Eighty-three women experienced spontaneous labor onset and five were undelivered at $41+5$ weeks.

The total rate of labor induction did not significantly differ between observation and acupuncture group (20\% versus $17 \%$ ). Moreover no differences were found as far as the indications to induce labor, in particular "prolonged pregnancy" was similar between groups (8/96 versus 5/99).

Observation and acupuncture groups showed a similar rate of pre-labor rupture of membranes (16\% versus $15.3 \%$ ), gestational age at delivery $(287.3 \pm 3$ versus $287.6 \pm 2.7$ days), birth weight $(3566 \pm 371.2$ versus $3540 \pm 384 \mathrm{~g})$, and rate of caesarean section $(18.8 \%$ versus $15.3 \%$ ).

To investigate between-group differences in time elapsed between inclusion and delivery, survival analysis was performed excluding women requiring labor induction: women

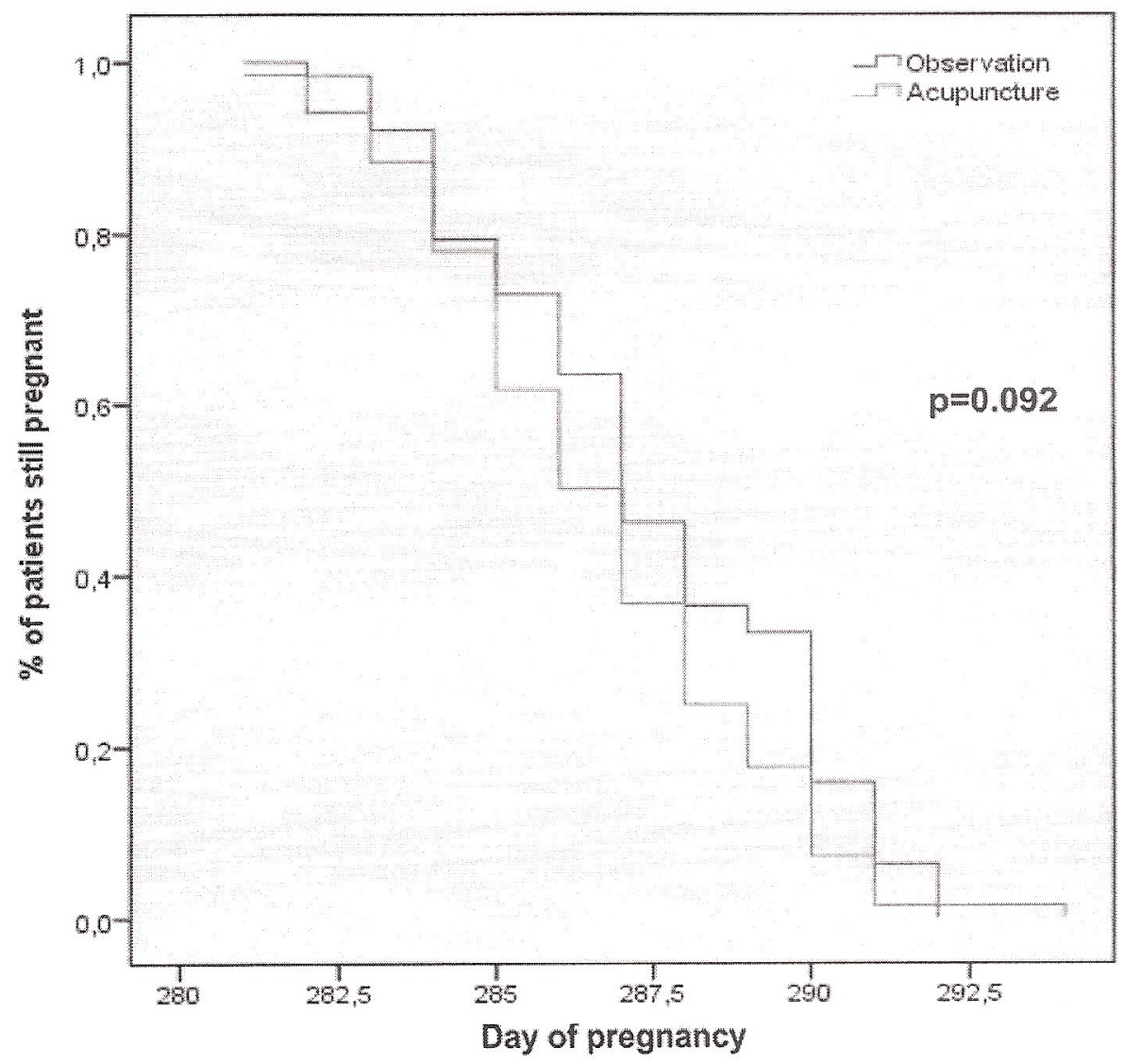

Figure 3. Survival analysis concerning between-group differences in time elapsed between inclusion and spontaneous delivery. 


\section{FIRST STAGE OF LABOUR}

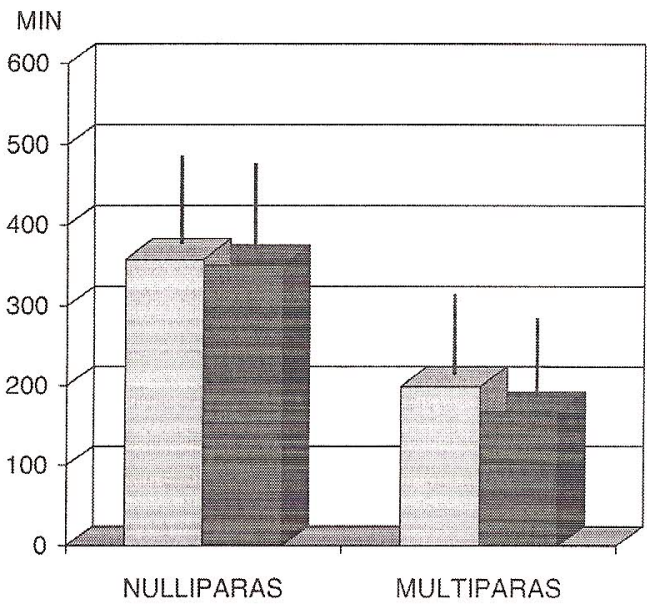

Figure 4. Duration of first stage in observation (white bars) and acupuncture group (black bars) in both nulliparous and multiparous women.

\section{SECOND STAGE OF LABOUR}

MIN

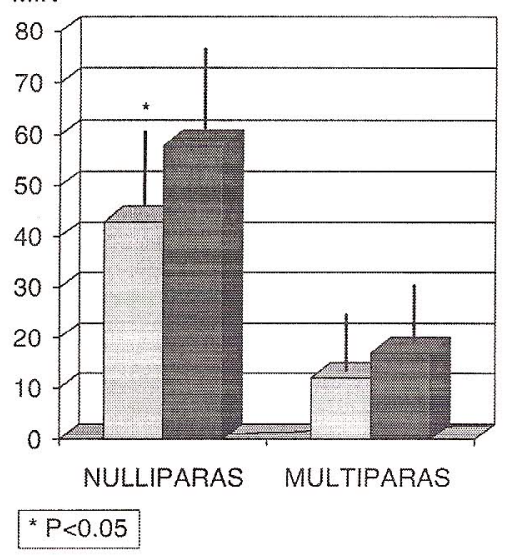

Figure 5. Duration of the second stage in observation (white bars) and acupuncture group (black bars) in both nulliparous and multiparous women. Asterisk *indicates a $p$ value lower than 0.05 .

receiving acupuncture showed a trend to deliver earlier than women in the observation group $[p<0.09]$ (Figure 3).

The length of labor stages was also evaluated. No differences were observed between the observation and acupuncture groups as far as the duration of the first stage of labor (Figure 4). Nulliparous women receiving acupuncture showed a significantly longer second stage when compared to those in observation group $(58.2+30$ versus $43.14 \pm 26.3$ minutes; $p<0.05$ ) despite the similar rate of occiput posterior position (Figure 5).

\section{Conclusion}

This study investigates the effects of acupuncture applied one week before planned induction in post-date pregnancy. Treatment did not modify the rate of labor induction performed for prolonged pregnancy at $41+5$ weeks.
Table 1. The features of the mentioned studies.

\begin{tabular}{|c|c|c|c|}
\hline & $\begin{array}{c}\text { Treatment } \\
\text { onset } \\
\text { (week) }\end{array}$ & $\begin{array}{c}\text { Treatment } \\
\text { duration }\end{array}$ & Acupoints \\
\hline Harper [7] & 40 & Every $3 / 4$ days & 6 Spleen \\
\hline Smith [8] & 41 & $\begin{array}{l}2 / 3 \text { days before } \\
\text { planned induction } \\
\text { at } 41+3\end{array}$ & $\begin{array}{l}\text { 31-32 Bladder } \\
6 \text { Spleen } \\
31 \text { - } 32 \text { Bladder } \\
4 \text { Large Intestine } \\
36 \text { Stomach } \\
3 \text { Liver }\end{array}$ \\
\hline Asher [9] & 38 & $\begin{array}{l}\text { Up to a maximum } \\
\text { of five treatments } \\
\text { over a } 2 \text {-weeks } \\
\text { period }\end{array}$ & $\begin{array}{l}6 \text { Spleen } \\
\text { 32-54 Bladder } \\
4 \text { Large Intestine }\end{array}$ \\
\hline Modlock [10] & $41+6$ & $\begin{array}{l}\text { Twice at the } 41+6 \\
\text { week }\end{array}$ & $\begin{array}{l}6 \text { Spleen } \\
4 \text { Large Intestine } \\
67 \text { Gold Bladder } \\
20 \text { Conception vessel }\end{array}$ \\
\hline Present Study & $40+4$ & $\begin{array}{l}\text { Every odd day until } \\
\text { planned induction } \\
\text { at } 41+5\end{array}$ & $\begin{array}{l}6 \text { Spleen } \\
34 \text { Gold Bladder } \\
4 \text { Large Intestine } \\
36 \text { Stomach } \\
3 \text { Liver }\end{array}$ \\
\hline
\end{tabular}

Now voltage electrical stimulation.

However, women receiving acupuncture showed a trend to deliver earlier than the observation group.

Our results agree with previous studies reporting a weak effect of acupuncture in the induction of labor onset. A randomized controlled study investigated the effects of electro-acupuncture applied for three or four consecutive days starting at 40 th week. In this clinical setting acupuncture showed a trend to reduce the rate of labor induction, the latency (time elapsed between enrolment and delivery), and the length of the labor stages in respect to observation, although statistical significance was not reached [7].

A further large randomized study compared true to sham acupuncture applied for two/three consecutive days before labor induction planned at $41+3$ weeks. The rate of labor induction and the length of labor stages do not differed between groups [8].

Asher et al. proposed pre-labor acupuncture starting at the $38^{\text {th }}$ week in order to evaluate the latency, the rate of spontaneous labor onset, and both maternal and neonatal outcomes. True acupuncture was compared either with sham acupuncture or observation. True acupuncture did not affect any of the above reported outcomes [9].

Recently a randomized study compared true and sham acupuncture applied twice at the $41+6$ week. In such clinical setting true acupuncture seems unable to induce labor onset within 24 hours from the first stimulation [10].

The present study demonstrated that acupuncture applied every odd day for one week seems ineffective in reducing the rate of labor induction performed for prolonged pregnancy at $41+5$ weeks. Previous reports reached similar conclusions, independently of the different timing, duration and mode of stimuli application (Table 1).

Moreover, in nulliparous women receiving acupuncture, we found a longer second stage of labor with respect to those receiving observation. Previous studies investigating such issue reached discrepant conclusions. Two of them 
DOI: $10.3109 / 14767058.2013 .845158$

demonstrated an effect of acupuncture to shorten the length of the first stage of labor and also to reduce the need of oxytocin augmentation [11,12]. Conversely, another report [13] found that acupuncture prolonged the second stage of labor, as in our series.

Biochemical studies suggest that acupuncture stimulates central oxytocin release and parasympathetic uterine activity increasing contractility $[11,14,15]$ but clinical observations seem not confirm such an effect. However, heterogeneity of treatments [timing, mode, duration] do not allow to reach definitive conclusions.

\section{Declaration of interest}

The authors report no conflicts of interest.

\section{References}

1. Gulmezoglu AM, Crowther CA, Middleton P. Induction of labour for improving birth outcomes for women at or beyond term. Cochrane Syst Rev 2006;4:CD004945 Review.

2. American College of Obstetrics and Gynccology: induction of labour. Practice Bullettin no 10; 1999.

3. Alfirevic Z, Kelly AJ, Dowswell T. Intravenous oxytocin alone for cervical ripening and induction of labour. Cochrane Database Syst Rev 2009; 7:CD003246.

4. Kelly AJ, Malik S, Smith L, et al, Vaginal prostaglandin (PGE2 and PGF2a) for induction of labour at term. Cochrane Database Syst Rev 2009;4:CD003101.
5. Maciocia $G$, ed. "Obstetrics and Gynecology in Chinese Medicine", China: Churchil Livingstone; 2002.

6. Smith CA. Crowther CA. Acupuncture for induction of labour. Cochrane Syst Rev 2012;7:CD002962 Review.

7. Harper TC, Coeytaux RR, Chen W, et al. A randomized controlled trial of acupuncture for initiation of labour in nulliparous women. J Mat Fetal Neonat Med 2006:19:465-70.

8. Smith CA, Crowther CA, Collins CT, Coyle ME. Acupuncture to induce labor: a randomized controlled trial. Obstet Gynecol 2008; 112:1067-74.

9. Asher GN, Coeytaux RR, Chen W, et al. Acupuncture to initiate labor (Acumons 2): a randomized, sham-controlled clinical trial $\mathrm{J}$ Mat Fetal Neonatal Med 2009:22:843-8.

10. Modlock J, Nielsen BB, Uldbjerg N. Acupuncture for the induction of labour: a double-blind randomised controlled study. Br $\int$ Obstet Gynecol 2010;117:1255-61.

11. Tempfer C, Zeisler H, Heinzl H, et al. Influence of Acupuncture on maternal serum levels of Interleukin-8, Prostaglandin F2alpha and beta-endorphin: a matched pair study. Obstet Gynecol 1998;92: 245-8.

12. Zeisler $H$, Tempfer $C$, Mayerhofer $K$, et al, Influence of Acupuncture on duration of labor. Gynecol Obstet Invest 1998 $46: 22-5$.

13. Lyrenas $S$, Lutsch $H$, Hetta $J$, Lindberg B. Acupuncture before Delivery: effect on labor. Gynecol Obstet Invest 1987;24: $217-24$.

14. Liao Y, Seto K, Saito $H$, et al. Effect of acupuncture on adrenocortical homone production. Am J Chin Med 1979;7: $362-71$.

15. Dunn PA, Rogers $D$, Halford $K$. Transcutaneous electrical nerve stinulation at acupuncture points in the induction of uterine contractions. Obstet Gynecol 1989;73:286-90. 